Marc Witzel *1981

Studium Erziehungswissenschaft, seit 2007 wissenschaftlicher Angestellter am Lehrstuhl Sozialpädagogik mit dem Schwerpunkt Handlungsfelder und Institutionen am Institut für Sozialpädagogik, Erwachsenenbildung und Pädagogik der frühen Kindheit an der Technischen Universität Dortmund.

Email: marc.witzel@tu-dortmund.de

\title{
Mediatisierung als Perspektive Sozialer Arbeit
}

\author{
Ein Systematisierungsversuch
}

\begin{abstract}
Obwohl sowohl Adressatinnen und Adressaten ebenso wie Professionelle der Sozialen Arbeit in ihren Lebenswelten selbstverständlich digitale Medien zur Kommunikation nutzen, werden Medien in der Sozialen Arbeit bisher als ein Randthema betrachtet. Mithilfe eines hier vorgelegten Systematisierungsversuchs soll die Komplexität des Themas deutlich gemacht und ein Plädoyer für eine sozialpädagogische Perspektive auf Mediatisierungsprozesse entfaltet werden.
\end{abstract}

\section{Keywords}

Medien, Mediatisierung, Systematik,

\section{Teaser}

Die Verbreitung sogenannter neuer oder digitaler Medien stellt auch für die Soziale Arbeit kein Randphänomen mehr dar. Für den Umgang mit Medien in den Alltagswelten von Adressatinnen und Adressaten, sowie Professionellen ist eine sozialpädagogische Perspektive auf diese Entwicklung notwendig.

\section{Einleitung}

Professionelle und Adressatinnen und Adressaten Sozialer Arbeit handeln mit und durch Medien und sind mit dem Medienhandeln anderer konfrontiert. Dabei geht es nicht nur um konkrete professionelle Arbeitsbezüge oder Formen des Medienhandelns, die als riskant oder förderlich eingestuft werden. Vielmehr werden durch das Medienhandeln Arbeits- und Lebensvollzüge intensiv geprägt. Während individuelle Auseinandersetzungen mit der Veränderung der Kommunikation sowohl von Professionellen, als auch von Adressatinnen und Adressaten heute allgegenwärtig sind, steht eine systematische Auseinandersetzung innerhalb der Sozialen Arbeit erst am Anfang. Mediatisierung als Meta-Prozess (1) wurde innerhalb der Sozialen Arbeit bisher unterschiedlich aufgegriffen und diskutiert (2). Dennoch ist das Themenfeld 'Mediatisierung' als komplexes und heterogenes Feld (3) scheinbar noch immer randständig innerhalb der Diskurse Sozialer Arbeit. Welche Perspektiven auf Mediatisierung innerhalb der Sozialen Arbeit möglich sind und warum Mediatisierung eine 
relevante Perspektive für die Soziale Arbeit ist (4), soll hier aufgezeigt werden.

\section{Mediatisierung als Meta-Prozess}

Mediatisierung als ein gesellschaftlicher Meta-Prozess zeichnet sich dadurch aus, dass eine zunehmende Zahl sozialer Handlungen von immer mehr Akteurinnen und Akteuren in medialen Bezügen vollzogen werden (vgl. Theunert \& Schorb 2010, S. 245; Krotz 2007, S. 278; Krotz 2012, S. 37). Meta-Prozesse sind gesellschaftliche Wandlungsprozesse, die im Unterschied zu Prozessen zeitlich nicht klar einzugrenzen sind und kulturübergreifend stattfinden (vgl. Krotz 2007, S. 27; zu Prozessen Miebach 2008). Als solch ein Meta-Prozess bezieht sich Mediatisierung zum einen auf andere Meta-Prozesse wie Globalisierung oder Individualisierung, thematisiert als Diskurs jedoch medienbezogene gesellschaftliche Veränderungen.

Der von Krotz (2012) genutzte Medienbegriff beschreibt Medien dabei als „[...] Entitäten [...], die dazu dienen, Kommunikation zu ermöglichen, zu modifizieren und zu gestalten [...]" (Krotz 2012, S. 41f). Medien gehen dabei insofern über Techniken hinaus, als dass Medien situativ und strukturell wirksam sind und nicht nur Kanäle für Kommunikationsinhalte darstellen, sondern die Produktion von Kommunikation selbst mitbestimmen.

Mediatisierung bezieht sich somit auf Kommunikationsprozesse von Akteurinnen und Akteuren. Kommunikation als soziales Handeln kann dabei zwischen Menschen medial vermittelt stattfinden, ebenso kann sie jedoch zwischen Menschen und allgemein adressierten, standardisierten Kommunikanten (wie Zeitungsartikeln, TV-Sendungen oder Webseiten (vgl. zu "Kommunikat" detailierter Zhao 2008, S. 90ff) erfolgen oder zwischen Menschen und interaktiven Systemen (vgl. Krotz, 2007 S. 57ff). Jegliche Form der Kommunikation als soziale Handlung unterliegt dabei sowohl einer situativen Ausgestaltung, als auch einer strukturellen Wirkung des Mediatisierungsprozesses.

Die situative Ausgestaltung bezieht sich dabei auf Medien als Erlebnisraum und Inszenierungsapparat im Kontext des Medienhandelns. Als kulturelle und soziale Erlebnisräume beziehen Medien den Menschen ein und gehen über das Übermitteln einer Nachricht hinaus. Es wird darüber hinaus ein eigenes, situatives Erlebnis im Medienhandeln geschaffen.

Als Inszenierungsapparat verweisen Medien darauf, dass Inhalte medienspezifischen "Kodier- und Produktionsweisen“ (Krotz 2012, S. 43) unterliegen und so medienspezifisch gestaltet und präsentiert werden. Auf diese Weise wird Kommunikation situativ ausgestaltet und differenziert. Auf einer strukturellen Ebene beschreibt der Mediatisierungsprozess zum einen die unterschiedlichen technischen Voraussetzungen für das Medienhandeln, die zwar die Verfügungsmöglichkeiten über diese Techniken und Kompetenzen zur Nutzung beinhalten, sich jedoch ebenso auf Infrastruktur beziehen. Zum anderen beschreibt er Medien als Institutionen, die in den alltäglichen Bezügen ihrer Nutzer kaum noch reflektiert werden, sondern die als bekannt vorausgesetzt werden (vgl. Krotz 2012, S. 43).

Die stetig zunehmende Internetnutzung als ein Element dieses Mediatisierungsprozesses verändert einerseits die Kommunikation und den Alltag von Akteurinnen und Akteuren und stellt in diesem Zusammenhang auch die Frage nach deren gesellschaftlicher Verortung neu (vgl. van Eimeren \& Frees 2012; Zillien 2006). Mediatisierungsprozesse sind daher sowohl auf der Mikroebene individuellen Handelns zu verorten, als auch auf der Makroebene 
gesellschaftlicher Veränderungen. Als Meta-Prozess gesellschaftlicher Veränderung verweist die Mediatisierungsthese darauf, dass die Veränderungen alltäglicher sozialer Kommunikationshandlungen sich auf die Kultur und Identität der Menschen ebenso auswirkt, wie auf deren Alltag und auf gesellschaftliche Strukturen (vgl. Krotz 2007, S. 56).

\section{Mediatisierung innerhalb der Sozialen Arbeit}

Obwohl in den letzten Jahren die Auseinandersetzung mit dem Thema Mediatisierung Sozialer Arbeit zugenommen hat, bleiben auch weiterhin viele Fragen offen.

Die Diskurse um Mediatisierung lassen sich in der Sozialen Arbeit anhand des Systematisierungsvorschlags von Flösser (1994) mithilfe der drei Dimensionen Profession, Organisation und Adressatinnen und Adressaten analytisch differenzieren. Dabei werden nicht nur die unterschiedlichen Dimensionen selbst in den Blick genommen, sondern darüber hinaus auch deren Zusammenspiel untereinander.

Für die Dimension der Profession werden im Kontext Sozialer Arbeit vor allem die Kompetenzen und Qualifikationen der Professionellen thematisiert. So gibt es eine Auseinandersetzung über die notwendigen Qualifikationen von Professionellen in Handlungsfeldern die medial vermittelte soziale Dienstleistungen erbringen, wie Onlineberatung (vgl. Kühne 2009), ebenso wie um Kompetenzen bezüglich der Kommunikation mit Adressaten und der Öffentlichkeit mittels digitaler Medien (vgl. Alfert \& Roggenbach 2012; Beringer 2012; Mishna, Bogo, Root, Sawyer \& Khoury-Kassabri 2012). Hinzu kommen Fragen digitaler Professionalität im Umgang mit computergestützen Arbeitsweisen bei der Erbringung sozialer Dienstleistungen (vgl. Ley 2012; Ley 2010). es Weiteren gibt es Auseinandersetzungen, was Professionelle können und wissen müssen, um angemessen mit den Medienhandlungsweisen von Adressatinnen und Adressaten umzugehen (vgl. Klein, Kutscher, Lojewski \& Schäfer 2009).

Für die Dimension der Organisation werden unterschiedlichste Themen aufgegriffen. Ein Thema ist der Umgang mit Medienangeboten. Dabei wird die grundsätzliche Frage nach der Nutzung von Medienangeboten durch die Organisationen gestellt. Inwiefern sollen Organisationen nicht nur mit einer eigenen Homepage, sondern auch innerhalb weiterer Medienangebote ihre Außendarstellung vornehmen? Damit verknüpft sind Auseinandersetzungen um Social-Media-Policies, die den Umgang der Organisationen und der in ihnen tätigen Akteurinnen und Akteure mit diesen Angeboten regeln. Dazu gehören die Auseinandersetzungen sowohl um Inhalte, welche über die unterschiedlichen Medienangebote kommuniziert werden, als auch um die Erreichbarkeit der Organisation und des Datenschutzes. Insbesondere Auseinandersetzungen um Datenschutz und der Erreichbarkeit von Organisationen (und auch von konkreten Professionellen) werden zudem an der Schnittstelle von Organisation und Adressatinnen und Adressaten aufgegriffen (vgl. Alfert, 2013; Eisfeld-Reschke \& Hölderle 2010).

Ein weiterer Bereich der organisationsbezogenen Überlegungen im Rahmen der Mediatisierung Sozialer Arbeit bezieht sich auf organisationsbezogene Abläufe. Sowohl interne Kommunikationsabläufe, als auch die Anforderungen unterschiedlichster Dokumentations- und Berichtspflichten werden im Rahmen von Mediatisierungsprozessen der Sozialen Arbeit thematisiert, wobei zumeist Standardisierungen und deren Folgen in den Fokus geraten. Im Zusammenspiel von Organisation und Profession werden zudem Überlegungen zur Re-Organisation von Arbeitsweisen sowie professionellen Handlungs- und 
Deutungsmustern relevant (vgl. Kutscher, Ley \& Seelmeyer 2011).

Für die Dimension der Adressatinnen und Adressaten lassen sich mindestens drei Stränge der Auseinandersetzung differenzieren, die den Mediatisierungsprozess aufgreifen. Zum einen werden die Adressatinnen und Adressaten medial erbrachter sozialer Dienstleistungen wie der Onlineberatung in den Blick genommen (vgl. Klein 2007). Innerhalb dieses Stranges werden sowohl die Anliegen der Adressatinnen und Adressaten, als auch deren spezifische sozioökonomische Situation betrachtet. Zudem geraten die Schnittstellen zu der Dimension der Professionellen und den konkreten Unteraktionssituationen zwischen Professionellen und Adressatinnen und Adressaten näher in den Blick.

In einem zweiten Strang werden die Möglichkeiten medienpädagogischer Arbeit innerhalb sozialpädagogischer Angebote analysiert. In erster Linie wird dies für Angebote der Kinderund Jugendarbeit thematisiert. Hier werden Möglichkeiten medienpädagogischer Projekte, notwendige Ausstattungen und die (angenommenen) Fähigkeiten der Adressatinnen und Adressaten thematisiert (vgl. Klein et al.. 2009).

Der dritte Strang richtet den Blick auf das Medienhandeln der Adressatinnen und Adressaten. Diese Analysen beziehen sich zumeist auf das Medienhandeln spezifischer Adressatinnen- und Adressatengruppen (z.B. Jugendliche oder Senioren). Innerhalb dieses Stranges werden zumeist umfassende Formen des Medienhandelns thematisiert, die als förderlich oder gefährdend diskutiert werden (vgl. Cleppien \& Scholz 2010).

Ein Querschnittsthema dieser Diskurse stellt das Thema digitale Ungleichheit dar. Die Reproduktion sozialer Ungleichheiten im Medienhandeln von Adressatinnen und Adressaten wird sowohl als Frage von Zugängen, als Reflektionsfrage sozialpädagogischer Professionalität, wie auch als Frage von Organisationshandeln aufgegriffen. Dabei werden sowohl Zugänge auf der Ebene der Ausstattung wie auch unterschiedliche Formen des Medienhandelns unter Berücksichtigung sozioökonomischer Lebenssituationen thematisiert (vgl. Witzel 2012).

Die hier aufgeführten Diskurse sind an dieser Stelle nur benannt und keineswegs als abgeschlossene Übersicht $\mathrm{zu}$ verstehen. Vielmehr soll mit diesem Systematisierungsbestrebungen ein Überblick über unterschiedliche Formen der Thematisierung von Mediatisierung ermöglicht werden. Ausgelassen ist hierbei die Differenzierung zwischen disziplinären und professionellen Diskursen, die innerhalb dieser Systematik eine weitere Differenzierung der Perspektiven ermöglicht.

\section{Mediatisierung als heterogenes und komplexes Feld}

Bietet sich die hier vorgenommene Differenzierung anhand der Dimensionen Profession, Organisation und Adressatinnen und Adressaten für eine retrospektive Differenzierung der unterschiedlichen Perspektiven an, so wird diese dem Phänomen der Mediatisierung und seiner Komplexität keineswegs gerecht. Zwangsläufig werden im Rahmen einer solchen Differenzierung relevante Zusammenhänge eines Meta-Prozesses Mediatisierung ausgeblendet.

Kutscher, Ley und Seelmeyer (2011) haben mit einem erweiterten Analysemodell auf diese Reduktion der Komplexität reagiert. Basierend auf den Dimensionen Profession, Organisation und Adressatinnen und Adressaten haben sie eine Heuristik entwickelt, in der sie von Aneignungsprozessen der vier Dimensionen Technik, Gegenständen, Subjekt sowie 
sozialer Kontext und struktureller Rahmen ausgehen. Mit dieser Heuristik werden sowohl Mikro- wie auch Makroprozesse des Medienhandelns in ihrer Interdependenz erfassbar.

Technik umfasst dabei die "Trägermedien" (vgl. Kutscher et al. 2011, S. 196) mit den in ihnen eingelagerten Formen der Sinnkonstruktion. Diese Sinnkonstruktionen sind dabei auf Wirkungen ausgerichtet, die erzielt werden sollen. In Medien wird mittels Techniken eine spezifische Struktur eingelagert, welche deren Nutzung vorstrukturiert. Eine solche Vorstrukturierung ist dennoch auf einen Aneignungsprozess durch die handelnden Subjekte angewiesen, der sich als "Gestaltungszwang" (Kutscher et al 2011, S. 197) einerseits und "Aneignungsspielraum" (Kutscher et al. 2011, S. 196) andererseits darstellt.

Die jeweiligen Gegenstände Sozialer Arbeit stellen die Inhalte dar, die in technische Formen überführt werden. Hierzu gehört z. B. die Falldokumentation, die in eine spezifische Software übersetzt wird. Dieser Prozess der Überführung von Gegenständen in technische Formen gestaltet die Gegenstände dabei neu, indem bestimmte Handlungen ermöglicht und andere ausgeschlossen werden.

Diese Gegenstände werden in ihrer technischen Form von den jeweiligen Subjekten angeeignet. Diese Aneignungsprozesse werden dabei beeinflusst von habitualisierten Zugangsweisen, sozioökonomischer Einbindung und Nutzungszielen. Dabei ist keineswegs davon auszugehen, dass diese jeweils zu gleich verlaufenden Aneignungsprozessen oder Ergebnissen von Aneignungsprozessen führen. Vielmehr wird ein eigener Rahmen für Handlungen im Kontext technischer Formen sozialpädagogischer Gegenstände entwickelt, der über die vermeintlichen festen technischen Formen hinausgehen kann. Dabei können Medien anders genutzt werden, als ursprünglich intendiert oder es können neue Handlungsformen aus den Aneignungsprozessen resultieren (vgl. Kutscher et al. 2011, S. 197ff).

Der soziale Kontext und strukturelle Rahmen der Aneignung beeinflusst diese ebenfalls. Den sozialen Kontext und strukturellen Rahmen bilden die organisationalen Erbringungskontexte sozialer Dienstleistungen ebenso wie die jeweiligen Ressourcen, die sowohl im Sinne von Arbeitskulturen, Organisationsstrukturen und sozialen Bezügen die Aneignungsprozesse beeinflussen (vgl. Kutscher et al. 2011, S. 196ff). Die zwischen den unterschiedlichen Dimensionen Technik, Gegenstände, Subjekt und sozialer Kontext und struktureller Rahmen stattfindenden Aneignungsprozesse machen als Fokuspunkt der Analyse somit ein je spezifisches Medienhandeln sichtbar, welches die Komplexität von Mediatisierungsprozessen noch einmal verdeutlicht.

\section{Mediatisierung als relevante Perspektive Sozialer Arbeit}

Anhand der hier dargestellten Perspektiven auf Mediatisierung als Meta-Prozess, im Rahmen der Dimensionen Profession, Organisation und Adressatinnen und Adressaten sowie als Aneignungsprozesse der Dimensionen Technik, Gegenstände, Subjekt und sozialer Kontext und struktureller Rahmen werden zwei wesentliche Elemente eines Mediatisierungsprozess innerhalb der Sozialen Arbeit deutlich.

Zum einen ist Mediatisierung in unterschiedlichen Dimensionen und mit vielfältigen Facetten schon immer ein Element sozialer Handlungen. Mit zunehmenden Kommunikationsmöglichkeiten für Subjekte, die mit einer Verbreitung und Ausdifferenzierung von Geräten und Kommunikationswegen einher geht, steigen auch in 
einem durch Kommunikation geprägtem Arbeitsfeld die Anforderungen an die Beteiligten dieser Kommunikationsprozesse.

Zum anderen verweisen die unterschiedlichen Perspektiven darauf, dass Mediatisierung nicht eindimensional zu denken ist. Eine zunehmende Ausstattung mit Geräten, steigende Nutzungszeiten und eine Verlagerung von Kommunikationsprozessen auf Medien oder die Einführung von Dokumentations- und Feedbacksystemen müssen in ihrer Komplexität innerhalb eines Mediatisierungsprozesses betrachtet werden. Soziale Arbeit ist sowohl als Profession wie Disziplin gefordert, dieser Komplexität zu begegnen.

Eine solche Begegnung erfordert mindestens zwei Perspektiven.

Zum einen besteht auch weiterhin ein großer Bedarf an disziplinärer und professioneller Auseinandersetzung mit dem Mediatisierungsprozess als einem gesellschaftlichen MetaProzess. Hierfür ist eine kontinuierliche Auseinandersetzung auf Basis theoretisch und empirisch fundierter Analysen notwendig. Die Komplexität des Mediatisierungsprozesses erfordert dafür den Blick auf die unterschiedlichen Dimensionen als Mikroperspektive ebenso, wie auf die Soziale Arbeit als System (Makroperspektive).

Zum anderen müssen neben individuellen Auseinandersetzungen mit dem Mediatisierungsprozess systematisch die Folgen und Entwicklungen sowohl auf der Ebene der individuellen Lebenssituation von Adressatinnen und Adressaten sowie Professionellen, als auch im Erbringungskontext sozialer Dienstleistungen und der Gestaltung sozialpädagogischer und sozialpolitischer Rahmenbedingungen Sozialer Arbeit grundsätzlich mitgedacht werden.

Individuelle Auseinandersetzungen engagierter Einzelner mit veränderten Formen von Kommunikation können dabei lediglich den Einstieg bilden. Eine dezidiert sozialpädagogische Perspektive in Profession, Disziplin und Ausbildung auf Mediatisierung als gesellschaftlichem Wandlungsprozess zu entwickeln, ist eine der aktuellen Herausforderungen für die Soziale Arbeit.

\section{Literaturverzeichnis}

Alfert, Nicole, Roggenbach, Viola (2012). Pädagogische Beziehungen im Zeitalter von Facebook. Soziale Passagen, 4 (1), 39-57. doi:10.1007/s12592-012-0094-2

Alfert, Nicole (2013). Facebook als Handlungsraum in der Jugendarbeit - Krise oder strategische Chance für Institutionen? In Karin Böllert, Nicole Alfert, Mark Humme (Hrsg.), Soziale Arbeit in der Krise (S. 85-107). Wiesbaden: Springer Fachmedien Wiesbaden.

Beringer, Barbara (2012). Der Nutzen von Facebook und Twitter fur die Sozialarbeit. Was Professionelle der Sozialen Arbeit im Umgang mit Social Media beachten sollten. Sozial Aktuell (9), 14-18.

Cleppien, Georg, Scholz, Detlef (2010). Exzessive Mediennutzung soziales Problem, Konflikt, Abhängigkeit. In Georg Cleppien, Ulrike Lerche (Hrsg.), Soziale Arbeit und Medien (S. 129151). Wiesbaden: VS Verlag für Sozialwissenschaften.

Eisfeld-Reschke, Jörg, Hölderle, Jona (2010). Social Media Policy für NonprofitOrganisationen. In 20 Schritten zum Leitfaden für den Umgang mit sozialen Medien: ikosom UG. 
Flösser, Gaby (1994). Soziale Arbeit jenseits der Bürokratie. Über das Management des Sozialen. Neuwied: Luchterhand.

Klein, Alexandra (2007). Soziales Kapital Online. Soziale Unterstützung im Internet. Eine Rekonstruktion virtualisierter Formen sozialer Ungleichheit. Dissertation, Universität Bielefeld. Bielefeld. Zugegriffen 22.08.2011. Verfügbar unter https://pub.unibielefeld.de/luur/download?func=downloadFile\&recordOld=2301811\&fileOld=2301814.

Klein, Alexandra, Kutscher, Nadia, Lojewski, Johanna, Schäfer, Miriam (2009). Medienkompetenzförderung für Kinder und Jugendliche in benachteiligten Lebenslagen. Konzept zur inhaltlichen, didaktischen und strukturellen Ausrichtung der mediepädagogischen Praxis in der Kinder und Jugendarbeit (LfM-Dokumentation, Bd. 36). Düsseldorf.

Krotz, Friedrich (2007). Mediatisierung. Fallstudien zum Wandel der Kommunikation. Wiesbaden: VS Verlag für Sozialwissenschaften.

Krotz, Friedrich (2012). Von der Entdeckung der Zentralperspektive zur Augmented Reality: Wie Mediatisierung funktioniert. In Friedrich Krotz, Andreas Hepp (Hrsg.), Mediatisierte Welten (S. 27-55). Wiesbaden: VS Verlag für Sozialwissenschaften.

Kühne, Stefan (2009). Aus- und Weiterbildung in der Online-Beratung. In S.tefan Kühne, Gerhard Hintenberger (Hrsg.), Handbuch Online-Beratung. Mit 9 Tabellen (S. 233-239). Göttingen: Vandenhoeck \& Ruprecht.

Kutscher, Nadia, Ley, Thomas, Seelmeyer, Udo (2011). Subjekt - Technik - Kontext. Zur Aneignung von Informations- und Kommunikationstechnologien in der Sozialen Arbeit. In Arbeitskreis "Jugendhilfe im Wandel" (Hrsg.), Jugendhilfeforschung. Kontroversen Transformationen - Adressierungen (S. 187-214). Wiesbaden: VS Verlag für Sozialwissenschaften.

Ley, Thomas (2010). "Unser Schreibzeug arbeitet mit an unseren Gedanken.". Oder: Zur Konstruktion des sozialpädagogischen Falles in computerisierten Arbeitsumgebungen. In Georg Cleppien \& Ulrike Lerche (Hrsg.), Soziale Arbeit und Medien (S. 219-233). Wiesbaden: VS Verlag für Sozialwissenschaften.

Ley, Thomas (2012). New Technologies for Practice. In Mel Gray, James Migley \& Sthephen A. Webb (Hrsg.), The Sage handbook of social work (S. 677-692). Los Angels: SAGE.

Miebach, Bernhard (2008). Prozess. In Nina Baur, Hermann Korte, Martina Löw \& Markus Schroer (Hrsg.), Handbuch Soziologie (S. 373-390). Wiesbaden: VS Verlag für Sozialwissenschaften, Wiesbaden.

Mishna, Faye, Bogo, Marion, Root, Jennifer, Sawyer, Jami-Leigh, Khoury-Kassabri, Mona (2012). "It just crept in": The Digital Age and Implications for Social Work Practice. Clinical Social Work Journal, 40 (3), 277-286. doi:10.1007/s10615-012-0383-4

Theunert, Helga \& Schorb, Bernd (2010). Sozialisation, Medienaneignung und Medienkompetenz in der mediatisierten Gesellschaft. In Maren Hartmann \& Andreas Hepp (Hrsg.), Die Mediatisierung der Alltagswelt (S. 243-254). Wiesbaden: VS Verlag für Sozialwissenschaften.

van Eimeren, Birgit \& Frees, Beate (2012). 76 Prozent der Deutschen online - neue Nutzungssituationen durch mobile Endgeräte. Ergebnisse der ARD/ZDF-Onlinestudie 2012. 
Media-Perspektiven (7-8), 362-379.

Witzel, Marc (2012). Medienhandeln, Digitale Ungleichheit und Distinktion. Medien + Erziehung, 56 (6), 81-92.

Zhao, Jin (2008). Interkulturalität von Textsortenkonventionen. Vergleich deutscher und chinesischer Kulturstile : Imagebroschüren (Forum für Fachsprachen-Forschung, Bd. 79). Berlin: Frank \& Timme.

Zillien, Nicole (2006). Digitale Ungleichheit. Neue Technologien und alte Ungleichheiten in der Informations- und Wissensgesellschaft (1. Aufl.). Wiesbaden: VS Verlag für Sozialwissenschaften. 